\title{
Regularization of subsolutions in discrete weak KAM theory
}

\author{
P. BERNARD, M. ZAVIDOVIQUE
}

July 18, 2021

\begin{abstract}
We expose different methods of regularizations of subsolutions in the context of discrete weak KAM theory. They allow to prove the existence and the density of $C^{1,1}$ subsolutions. Moreover, these subsolutions can be made strict and smooth outside of the Aubry set.
\end{abstract}

\section{Introduction}

We consider a smooth connected Riemannian manifold $M$ endowed with the distance $\mathrm{d}(.,$.$) coming$ from the Riemannian metric. Fixing a cost function $c: M \times M \rightarrow \mathbb{R}$ we study the functions $u: M \rightarrow \mathbb{R}$ which satisfy

$$
\forall(x, y) \in M \times M, \quad u(y)-u(x) \leqslant c(x, y),
$$

we call them subsolutions, by analogy with those appearing in Weak KAM theory (see [FS04, Ber07] for example). We will denote by $\mathcal{S S}$ the set of subsolutions, and by $\mathcal{S S}_{C}=\mathcal{S} \mathcal{S} \cap C^{0}(M, \mathbb{R})$ the set of continuous subsolutions. These subsolutions are one of the important objects in discrete (in time) weak KAM theory. Some other aspects of this discrete theory have been discussed in [Gom05, BB07, Zav12]. This theory is also closely related to the time-periodic weak KAM theory, discussed for example in [CISM00, Ber08] and many other papers. In many aspects, these various settings (discrete, time-periodic, autonomous) are similar, but differences appear for some specific questions. For example, the convergence of the Lax-Oleinik semi-group holds only in the autonomous setting, see [Fat98, FM00, Ber02, BR05], the Hamilton-Jacobi equation does not have such a nice form in the discrete setting as in the autonomous setting, see [Gom05]. Some other specific aspects of the discrete case are discussed in [Zav12]. Concerning the regularity of subsolutions, the existence of $C^{1}$ subsolutions was obtained in [Zav12] in the discrete setting by an adaptation of the original proof of Fathi and Siconolfi [FS04]. On the other hand, the proof of the existence of $C^{1,1}$ subsolutions given in [Ber07] for the autonomous setting does not extend to the discrete setting. The existence of $C^{1,1}$ subsolutions was however obtained in [Zav10] by a different method. Our goal here is to extend and simplify the results of this paper.

Defining, as usual, the discrete Lax-Oleinik operators

$$
T_{c}^{-} u(x)=\inf _{y \in M} u(y)+c(y, x), \quad T_{c}^{+} u(x)=\sup _{y \in M} u(y)-c(x, y)
$$

we see that a function $u$ is a subsolution if and only if one of the equivalent relations is verified:

$$
u \leqslant T_{c}^{-} u \quad \text { or } \quad T_{c}^{+} u \leqslant u
$$

Note as a consequence that the functions $T_{c}^{-} u$ and $T_{c}^{+} u$ are themselves subsolutions whenever $u$ is a subsolution. We will use the following hypothesis on $c$. More concrete hypotheses implying this one are given below.

Hypothesis 1. For each subsolution $u$, the functions $T_{c}^{-} u$ and $-T_{c}^{+} u$ are locally semiconcave ${ }^{1}$.

\footnotetext{
${ }^{1}$ Throughout the paper, we call semiconcave what is sometimes called semiconcave with a linear modulus.
} 
Subsolutions do not necessarily exist, and, when they exist, they are not necessarily continuous (the continuity of subsolutions is discussed in [Zav12]). Under Hypothesis 1, the existence of a continuous subsolution is implied by the existence of a (possibly discontinuous) subsolution $u$, just consider the subsolution $T_{c}^{-} u$, which is locally semiconcave hence locally Lipschitz. See also Lemma 2.2 below.

Theorem 1. If Hypothesis 1 holds, then the set of locally $C^{1,1}$ subsolutions is dense in the set of continuous subsolutions for the strong topology.

We recall that the strong (or Whitney) topology on $C^{0}(M, \mathbb{R})$ is induced by the basis of open sets:

$$
O_{\epsilon, f}=\left\{g \in C^{0}(M, \mathbb{R}), \quad \forall x \in M, \quad|f(x)-g(x)|<\epsilon(x)\right\}
$$

where $f \in C^{0}(M, \mathbb{R})$ and $\epsilon$ is a continuous positive valued function on $M$. For further precisions on this topology, see [Hir94, Chapter 2]. The existence of $C^{1,1}$ subsolutions was proved in [Zav10], but the density is new. In [Zav10], the existence of $C^{1,1}$ subsolutions is deduced from the following result of Ilmanen (see [Ilm93, Car01, FZ10, Ber10]):

Theorem 2. Let $f$ and $g$ be locally semiconcave functions on $M$ such that $f+g \geqslant 0$. Then there exists a locally $C^{1,1}$ function $u$ such that $-g \leqslant u \leqslant f$.

We will offer a direct proof of Theorem 1, which is inspired from the proof of Ilmanen's Lemma given in [Ber10]. Note that Theorem 1 implies Theorem 2. This follows immediately from the equivalence, for a given function $u$, between the two following properties:

- the function $g+u$ is bounded from below and $-g \leqslant u-\inf (g+u) \leqslant f$;

- the function $u$ is a subsolution for the $\operatorname{cost} c(x, y)=g(x)+f(y)$.

We need to introduce more definitions before we state our other results. The subsolution $u$ is called free at $x$ if

$$
T_{c}^{+} u(x)<u(x)<T_{c}^{-} u(x)
$$

We define the set $\mathcal{A}_{u}$ as

$$
\mathcal{A}_{u}:=\left\{x \in M, \quad T_{c}^{+} u(x)=u(x)=T_{c}^{-} u(x)\right\} \subset M,
$$

and the Aubry set $\mathcal{A}$ as

$$
\mathcal{A}:=\bigcap_{u \in \mathcal{S} \mathcal{S}} \mathcal{A}_{u} \subset M
$$

where the intersection is taken on all subsolutions. Under hypothesis 1 , the sets $\mathcal{A}_{u}$ are closed, since they are defined by the equality $T_{c}^{+} u=T_{c}^{-} u$. The set $\mathcal{A}$ is then also closed. Moreover, it makes no difference to restrict the intersection to continuous subsolutions in the definition of $\mathcal{A}$, by Lemma 2.2 below. We say that the subsolution $u$ is strict at $(x, y)$ if

$$
u(y)-u(x)<c(x, y) .
$$

Obviously, the subsolution $u$ is strict at $(x, y)$ and at $(y, x)$ for each $y$ if it is free at $x$. We define the set

$$
\overline{\mathcal{A}}_{u}:=\left\{(x, y) \in M^{2}: u(y)-u(x)=c(x, y)\right\}
$$

We also define

$$
\overline{\mathcal{A}}:=\bigcap_{u \in \mathcal{S} \mathcal{S}} \overline{\mathcal{A}}_{u}
$$

where the intersection is taken on all subsolutions. Equivalently, if Hypothesis 1 holds, the intersection can be taken on continuous subsolutions, by Lemma 2.2. This yields that $\overline{\mathcal{A}}$ is also closed.

Theorem 3. Assume that c satisfies Hypothesis 1. Given a subsolution u, there exists a subsolution $v$ such that 
- $v=u$ on $\mathcal{A}_{u}$,

- $v$ is smooth and free on the complement of $\mathcal{A}_{u}$,

- $v$ is locally $C^{1,1}$,

- $v$ is strict at each pair $(x, y)$ where $u$ is strict.

We can then obtain a subsolution which is as smooth, free, and strict as possible:

Theorem 4. If c satisfies hypothesis 1 and admits a subsolution, then there exists a locally $C^{1,1}$ subsolution which is free and smooth in the complement of $\mathcal{A}$, and strict on the complement of $\overline{\mathcal{A}}$.

Observe as a consequence that the projections of $\overline{\mathcal{A}}$ on both the first and the second factor are contained in $\mathcal{A}$, (and, under the additional hypothesis 2, each of these projections is equal to $\mathcal{A}$, see below). Strict $C^{1,1}$ subsolutions were obtained in [Zav10] under an additional twist assumption. We will use a simple trick of [Ber07] to obtain directly the general result from Theorem 1 . That the subsolutions can be made smooth outside of $\mathcal{A}$ is well-known. It will certainly not be a surprise to specialists that this can be done without destroying the global $C^{1,1}$ regularity, although we do not know any reference for this statement. We prove it using a regularization procedure due to De Rham [dR73]. This proof also applies to the "classical" (as opposed to discrete) weak KAM theory.

The abstract Hypothesis 1 holds in a more concrete setting, introduced in [Zav12]:

Hypothesis 2. The function $c$ satisfies the following properties:

- uniform super-linearity: for every $k \geqslant 0$, there exists $C(k) \in \mathbb{R}$ such that

$$
\forall(x, y) \in M \times M, \quad c(x, y) \geqslant k \mathrm{~d}(x, y)-C(k)
$$

- uniform boundedness: for every $R \in \mathbb{R}$, there exists $A(R) \in \mathbb{R}$ such that

$$
\forall(x, y) \in M \times M, \quad \mathrm{~d}(x, y) \leqslant R \Rightarrow c(x, y) \leqslant A(R)
$$

- local semiconcavity: for each point $\left(x_{0}, y_{0}\right)$ there is a domain of chart containing $\left(x_{0}, y_{0}\right)$ and a smooth function $f(x, y)$ such that $c-f$ is concave in the chart. (This holds for example if $c$ is $C^{2}$ or locally $\left.C^{1,1}\right)$.

This hypothesis has two important consequences, as was proved in [Zav12]. First, it implies Hypothesis 1. Second, it implies that the extrema in the definitions of $T_{c}^{ \pm} u(x)$ are reached for each continuous subsolution $u$ and each $x \in M$. This in turn implies that the the projection of $\overline{\mathcal{A}}$ on the first, as well as on the second, factor are equal to $\mathcal{A}$, which corresponds to the projected Aubry set introduced in [Zav12]:

Lemma 1.1. Assume that $c$ satisfies Hypothesis 2. Given $x \in \mathcal{A}$, there exist $y$ and $z$ such that $(x, z)$ and $(y, x)$ are in $\overline{\mathcal{A}}$.

Proof. Let $w$ be a continuous subsolution which is strict outside of $\overline{\mathcal{A}}$ (such a solution exists by Theorem 4). Let $y$ be such that $T_{c}^{-} w(x)=w(y)+c(y, x)$. Since $x \in \mathcal{A}$ we obtain that $w(x)-w(y)=c(y, x)$. Hence $(y, x) \in \overline{\mathcal{A}}_{w}=\overline{\mathcal{A}}$. The existence of $z$ is proved in the same way, using $T_{c}^{+}$.

Finally, let us mention one last setting in which Hypothesis 1 holds :

Hypothesis 3. The function $c$ is locally bi-semiconcave:

for all $(x, y) \in M \times M$ we can find the following:

- neighborhoods $U$ and $V$ of respectively $x$ and $y$,

- diffeomorphisms $\varphi_{1}$ and $\varphi_{2}$ from $B_{n}$ to respectively $U$ and $V\left(B_{n}\right.$ is the unit ball in $\left.\mathbb{R}^{n}\right)$, 
- smooth functions $f$ and $g$ from $B_{n}$ to $\mathbb{R}$,

such that for each $x \in M$, the function $z \mapsto c\left(x, \varphi_{2}(z)\right)-g(z)$ is concave and for all $y \in M$, the function $z \mapsto c\left(\varphi_{1}(z), y\right)-f(z)$ is concave.

It is easy to prove, as in [Zav10, Proposition 4.6], that Hypothesis 3 also implies Hypothesis 1 (using that an infimum of equi-semiconcave functions is itself semiconcave).

\section{Preliminaries}

We gather here some useful facts obtained from elementary manipulations of the Lax-Oleinik operators. Let us first list, without proof, some properties of the operators $T_{c}^{ \pm}$.

- Monotony : $u \leqslant v \Rightarrow T_{c}^{ \pm} u \leqslant T_{c}^{ \pm} v$.

- Convexity : Given a sequence $u_{n}$ of functions and a sequence $a_{n}$ of non-negative numbers such that $\sum_{n \in \mathbb{N}} a_{n}=1$, and such that the series $\sum_{n \in \mathbb{N}} a_{n} T_{c}^{-} u_{n}, \sum_{n \in \mathbb{N}} a_{n} u_{n}$ and $\sum_{n \in \mathbb{N}} a_{n} T_{c}^{+} u_{n}$ are converging point-wise, we have

$$
T_{c}^{-}\left(\sum_{n \in \mathbb{N}} a_{n} u_{n}\right) \geqslant \sum_{n \in \mathbb{N}} a_{n} T_{c}^{-} u_{n} \quad, \quad T_{c}^{+}\left(\sum_{n \in \mathbb{N}} a_{n} u_{n}\right) \leqslant \sum_{n \in \mathbb{N}} a_{n} T_{c}^{+} u_{n} .
$$

The set $\mathcal{S S}$ of subsolutions is convex, and it is closed under point-wise convergence. A convex combination $\sum_{n \in \mathbb{N}} a_{n} u_{n}$ of subsolutions, with a point-wise convergent sum, is a subsolution; it is free at $x$ (resp. strict at $(x, y))$ provided there exists $n$ such that $a_{n}>0$ and such that $u_{n}$ is free at $x$ (resp. strict at $(x, y))$.

- We have the equalities $T_{c}^{+} \circ T_{c}^{-} \circ T_{c}^{+}=T_{c}^{+}$and $T_{c}^{-} \circ T_{c}^{+} \circ T_{c}^{-}=T_{c}^{-}$.

- We have the inequalities

$$
T_{c}^{+} \circ T_{c}^{-} u \leqslant u \quad, \quad T_{c}^{-} \circ T_{c}^{+} u \geqslant u
$$

for each function $u$.

- If $u$ is a subsolution, then

$$
T_{c}^{+} u \leqslant T_{c}^{+} \circ T_{c}^{-} u \leqslant u \leqslant T_{c}^{-} \circ T_{c}^{+} u \leqslant T_{c}^{-} u
$$

The following criterion for subsolutions is taken from [Zav12]:

Lemma 2.1. Let $u$ be a subsolution and let us consider a function $v$ such that

$$
u \leqslant v \leqslant T_{c}^{-} u
$$

then $v$ itself is a subsolution.

Proof. The statement follows from the inequalities $u \leqslant v \leqslant T_{c}^{-} u \leqslant T_{c}^{-} v$.

Playing with the Lax-Oleinik operators also leads to:

Lemma 2.2. Let $u$ be a subsolution, then the subsolution

$$
v:=\frac{T_{c}^{+} u+T_{c}^{+} \circ T_{c}^{-} u+T_{c}^{-} \circ T_{c}^{+} u+T_{c}^{-} u}{4}
$$

is free on the complement of $\mathcal{A}_{u}$, equal to $u$ on $\mathcal{A}_{u}$, and strict on the complement of $\overline{\mathcal{A}}_{u}$. If Hypothesis 1 holds, then $v$ is locally Lipschitz. 
We then have $\mathcal{A}_{v} \subset \mathcal{A}_{u}$, but this inclusion is not necessarily an equality.

Proof. To prove that $v$ is free on the complement of $\mathcal{A}_{u}$, we consider a point $x$ at which $v$ is not free, and prove that $x \in \mathcal{A}_{u}$. We either have $T_{c}^{+} v(x)=v(x)$ or $T_{c}^{-} v(x)=v(x)$. In the first case, we have

$$
4 v(x)=4 T_{c}^{+} v(x) \leqslant T_{c}^{+} \circ T_{c}^{+} u(x)+T_{c}^{+} \circ T_{c}^{+} \circ T_{c}^{-} u(x)+T_{c}^{+} \circ T_{c}^{-} \circ T_{c}^{+} u(x)+T_{c}^{+} \circ T_{c}^{-} u(x)
$$

hence the inequalities

$$
\begin{array}{r}
T_{c}^{+} \circ T_{c}^{+} u(x) \leqslant T_{c}^{+} u(x) \quad, \quad T_{c}^{+} \circ T_{c}^{+} \circ T_{c}^{-} u(x) \leqslant T_{c}^{+} \circ T_{c}^{-} u(x) \\
T_{c}^{+} \circ T_{c}^{-} \circ T_{c}^{+} u(x)=T_{c}^{+} u(x) \leqslant T_{c}^{-} \circ T_{c}^{+} u(x) \quad, \quad T_{c}^{+} \circ T_{c}^{-} u(x) \leqslant T_{c}^{-} u(x)
\end{array}
$$

sum to an equality, hence they are equalities. In view of (1) the two last equalities imply that $T_{c}^{+} u(x)=u(x)=T_{c}^{-} u(x)$. The second case is similar. It then follows from Lemma 2.3 below that $v$ is strict outside of $\overline{\mathcal{A}}_{u}$.

The following Lemma allows to reduce strictness questions to freedom questions, and ends the proof of Lemma 2.2.

Lemma 2.3. Let $u, v$ be subsolutions, such that $v$ is free outside of $\mathcal{A}_{u}$ and equal to $u$ on $\mathcal{A}_{u}$, then $v$ is strict at each point $(x, y)$ where $u$ is strict.

Proof. Let $(x, y)$ be a pair at which $v$ is not strict. Then $v(y)-v(x)=c(x, y)$, hence $T_{c}^{-} v(y)=v(y)$ and $T_{c}^{+} v(x)=v(x)$. Since $v$ is free outside of $\mathcal{A}_{u}$, this implies that both $x$ and $y$ belong to $\mathcal{A}_{u}$. Since $u=v$ on $\mathcal{A}_{u}$, we conclude that

$$
u(y)-u(x)=v(y)-v(x)=c(x, y)
$$

hence $u$ is not strict at $(x, y)$.

It will also be useful to quantify the freedom of a subsolution $u$ by its leverage function:

Definition 2.4. The leverage function $\lambda_{u}: M \longrightarrow[0, \infty)$ of the subsolution $u$ is defined by:

$$
\lambda_{u}(x):=\frac{1}{3} \min \left(T_{c}^{-} u(x)-u(x), u(x)-T_{c}^{+} u(x)\right) .
$$

Note that $u$ is free at $x$ if and only if $\lambda_{u}(x)>0$.

Lemma 2.5. Let $u$ be a subsolution and let $v$ be another function such that $|u-v| \leqslant \lambda_{u}$, then $v$ is itself a subsolution. Moreover, if $u$ is free at $x$ then so is $v$, and if $u$ is strict at $(x, y)$, then so is $v$.

Proof. By definition, we have

$$
3 \max \left\{\lambda_{u}(x), \lambda_{u}(y)\right\} \leqslant \max \left\{u(x)-T_{c}^{+} u(x), T_{c}^{-} u(y)-u(y)\right\} \leqslant c(x, y)-u(y)+u(x) .
$$

We conclude that

$$
0 \leqslant \max \left\{\lambda_{u}(x), \lambda_{u}(y)\right\} \leqslant c(x, y)-v(y)+v(x)
$$

hence that

$$
T_{c}^{+} v(x)+\lambda_{u}(x) \leqslant v(x) \leqslant T_{c}^{-} v(x)-\lambda_{u}(x)
$$

for each $x$, which implies that $v$ is a subsolution which is free at points where $u$ is free. The last claim follows from Lemma 2.3. 


\section{The uniform case on $\mathbb{R}^{n}$ and the Jensen transforms}

In this section we work on $M=\mathbb{R}^{n}$. A function $u: \mathbb{R}^{n} \longrightarrow \mathbb{R}$ is called $k$-semiconcave if $u(x)-k\|x\|^{2}$ is concave. We make the following more quantitative version of Hypothesis 1 on the cost $c$ :

Hypothesis $\mathbf{1}-\mathbf{K}$. There exists a constant $K$ such that for each subsolution $u$, the functions $T_{c}^{-} u$ and $-T_{c}^{+} u$ are $K$-semiconcave.

One setting which implies this condition is tho following version of Hypothesis 3 :

Hypothesis $\mathbf{3}-\mathbf{K}$. There exists a constant $K$ such that the function $x \longmapsto c(x, y)$ is $K$-semiconcave for each $y$ and the function $y \longmapsto c(x, y)$ is $K$-semiconcave for each $x$.

We will use the Jensen transforms which associate, to a function $u: \mathbb{R}^{n} \rightarrow \mathbb{R}$ and a positive real number $t$, the functions

$$
J^{-t} u(x)=\inf _{y \in \mathbb{R}^{n}}\left(u(y)+\frac{1}{t}\|y-x\|^{2}\right), \quad J^{+t} u(x)=\sup _{y \in \mathbb{R}^{n}}\left(u(y)-\frac{1}{t}\|y-x\|^{2}\right) .
$$

These are nothing but the Lax-Oleinik operators associated to the $\operatorname{costs} c_{t}(x, y)=\frac{1}{t}\|y-x\|^{2}$.

Theorem 5. Let $u$ be a uniformly continuous subsolution. The function $J^{-t} \circ J^{+2 t} \circ J^{-t} u$ is finite and, for $t$ small enough, it is a $C^{1,1}$ subsolution. Moreover, it converges uniformly to $u$ as $t \rightarrow$ 0. More precisely, if $u$ is a uniformly continuous subsolution then for $t, s<K^{-1}$ the functions $J^{-t} \circ J^{+(t+s)} \circ J^{-s} u$ and $J^{+t} \circ J^{-(t+s)} \circ J^{+s} u$ are $C^{1,1}$ subsolutions which converge uniformly to $u$ as $t, s \rightarrow 0$. Moreover, we have

$$
T_{c}^{+} \circ T_{c}^{-} u \leqslant J^{-t} \circ J^{+(t+s)} \circ J^{-s} u \leqslant T_{c}^{-} u \quad, \quad T_{c}^{+} u \leqslant J^{+t} \circ J^{-(t+s)} \circ J^{+s} u \leqslant T_{c}^{-} \circ T_{c}^{+} u .
$$

Note that the last inequalities imply that $J^{-t} \circ J^{+(t+s)} \circ J^{-s} u$ and $J^{+t} \circ J^{-(t+s)} \circ J^{+s} u$ are subsolutions, by Lemma 2.1. We recall a few properties of the Jensen transforms, most of which are proved in [Ber10] or [AD00]. Both families of operators $J^{-}$and $J^{+}$are semi-groups. They are monotonous in the following way:

$$
\forall s>t>0, \quad \inf u \leqslant J^{-s} u \leqslant J^{-t} u \leqslant u \leqslant J^{+t} u \leqslant J^{+s} u \leqslant \sup u
$$

and in the following one:

$$
u \leqslant v \Rightarrow\left\{\forall t \geqslant 0, \quad J^{-t} u \leqslant J^{-t} v \text { and } J^{+t} u \leqslant J^{+t} v\right\} .
$$

We call modulus of continuity a continuous function $\rho:[0, \infty) \longrightarrow[0, \infty)$ such that $\rho(0)=0$. A function $f$ is said $\rho$-continuous if $|f(y)-f(x)| \leqslant \rho(\|y-x\|)$ for all $x$ and $y$. Given a modulus of continuity $\rho$, there exists a modulus of continuity $\epsilon$ such that, for each $\rho$-continuous function $u$, the following properties hold:

- the functions $J^{-t} u$ and $J^{+t} u$ are finite-valued and $\rho$-continuous for each $t \geqslant 0$,

- $J^{-t} u$ is $t^{-1}$-semiconcave and $J^{+t} u$ is $t^{-1}$-semiconvex,

- $\left\|J^{-t} u-u\right\|_{\infty}+\left\|J^{+t} u-u\right\|_{\infty} \leqslant \epsilon(t)$,

- $J^{-t} \circ J^{+t} u \geqslant u$ and $J^{+t} \circ J^{-t} u \leqslant u$,

- the equality $J^{-t} \circ J^{+t} u=u$ (resp. $J^{+t} \circ J^{-t} u=u$ ) holds if and only if $u$ is $t^{-1}$-semiconcave (resp. $t^{-1}$-semiconvex),

- if $u$ is semiconvex (resp. semiconcave) then $J^{-t} \circ J^{+t} u$ (resp. $J^{+t} \circ J^{-t} u$ ) is $C^{1,1}$ (and finite valued). 
Using these properties, we now prove Theorem 5. Let $u$ be a uniformly continuous subsolution, with modulus $\rho$. Since the function $u$ is a subsolution, we have $u \leqslant T_{c}^{-} u$ hence $T_{c}^{-} u$ is finite-valued. Our hypothesis is that the function $T_{c}^{-} u$ is $K$-semiconcave. For $s<K^{-1}$, we have

$$
u \leqslant J^{-s} \circ J^{+s} u \leqslant J^{-s} \circ J^{+s}\left(T_{c}^{-} u\right)=T_{c}^{-} u,
$$

where the last inequality follows from the $K$-semiconcavity of $T_{c}^{-} u$ and the properties of $J^{-} \circ J^{+}$listed above. We conclude that the function $J^{-s} \circ J^{+s} u$ is a $\rho$-continuous, $s^{-1}$-semiconcave subsolution. Similarly, if $u$ is $\rho$-continuous and $t<K^{-1}$, then the function $J^{+t} \circ J^{-t} u$ is a $\rho$-continuous, $t^{-1}$ semiconvex subsolution. Applying this observation to the function $J^{-s} \circ J^{+s} u$, we conclude that $J^{+t} \circ J^{-t} \circ J^{-s} \circ J^{+s} u$ is a $\rho$-continuous subsolution. This subsolution is $C^{1,1}$ since $J^{-s} \circ J^{+s} u$ is semiconcave. We have the inequality

$$
T_{c}^{+} \circ T_{c}^{-} u=J^{+t} \circ J^{-t}\left(T_{c}^{+} \circ T_{c}^{-} u\right) \leqslant J^{+t} \circ J^{-t} u \leqslant J^{+t} \circ J^{-t} \circ J^{-s} \circ J^{+s} u \leqslant J^{+t} \circ J^{-t}\left(T_{c}^{-} u\right) \leqslant T_{c}^{-} u .
$$

Finally, we have

$$
u \leqslant J^{-s} \circ J^{+s} u \leqslant J^{-s}\left(u+\left\|J^{+s} u-u\right\|_{\infty}\right) \leqslant\left\|J^{+s} u-u\right\|_{\infty}+\left\|J^{-s} u-u\right\|_{\infty}+u \leqslant u+\epsilon(s)
$$

and similarly $u-\epsilon(t) \leqslant J^{+t} \circ J^{-t} u \leqslant u$ hence

$$
u-\epsilon(t) \leqslant J^{+t} \circ J^{-t} u \leqslant J^{+t} \circ J^{-(t+s)} \circ J^{+s} u \leqslant J^{-s} \circ J^{+s} u \leqslant u+\epsilon(s),
$$

where $\epsilon$ is the modulus associated to $\rho$ in the list of properties of $J$.

\section{The general case}

In this section, we come back to the general setting and prove Theorem 1 . We derive it from the uniform version using partitions of unity, as was done in [Ber10] for Ilmanen's Lemma. We fix a locally finite atlas $\left(\phi_{i}\right)_{i \in I}$ constituted of smooth maps $\phi_{i}: B_{n} \rightarrow M$, where $B_{n}$ is the open unit ball. We assume that all the images $\phi_{i}\left(B_{n}\right)$, for $i \in I$, are relatively compact in $M$. Moreover, we consider a smooth partition of unity $\left(g_{i}\right)_{i \in I}$ subordinated to the locally finite open covering $\left(\phi_{i}\left(B_{n}\right)\right)_{i \in I}$. Given positive numbers $a_{i}, b_{i}, i \in I$, we define the operators

$$
\begin{aligned}
& \forall x \in M, \quad S u(x)=\sum_{i \in I}\left[J^{-a_{i}} \circ J^{+a_{i}}\left(g_{i} u \circ \phi_{i}\right)\right] \circ \phi_{i}^{-1}(x), \\
& \forall x \in M, \quad \check{S} u(x)=\sum_{i \in I}\left[J^{+b_{i}} \circ J^{-b_{i}}\left(g_{i} u \circ \phi_{i}\right)\right] \circ \phi_{i}^{-1}(x) .
\end{aligned}
$$

The functions in the sums are extended to the whole of $M$ by the value zero outside of the domain $\phi_{i}\left(B_{n}\right)$. The sums are locally finite hence well-defined. Theorem 1 follows from:

Theorem 6. Assume that the cost c satisfies Hypothesis 1. Let $u$ be a continuous subsolution and $\epsilon: M \rightarrow] 0, \infty)$ be a continuous function. For suitably chosen positive constants $\left(a_{i}\right)_{i \in I}$ and $\left(b_{i}\right)_{i \in I}$, the function $\check{S} \circ S(u)$ is a locally $C^{1,1}$ subsolution such that $|u-\check{S} \circ S u| \leqslant \epsilon$ and

$$
T_{c}^{+} \circ T_{c}^{-} u \leqslant \check{S} \circ S u \leqslant T_{c}^{-} u \text {. }
$$

Proof. Since the image $\phi_{i}\left(B_{n}\right)$ is relatively compact and since the atlas is locally finite the set $A_{i}=\left\{j \in I, \quad \phi_{j}\left(B_{n}\right) \cap \phi_{i}\left(B_{n}\right) \neq \varnothing\right\}$ is finite, let us denote by $e_{i}$ its cardinal. Setting

$$
\epsilon_{i}:=\frac{\min _{j \in A_{i}} \inf _{x \in B_{n}} \epsilon\left(\phi_{j}(x)\right)}{2 \max _{j \in A_{i}} e_{j}},
$$


we observe that

$$
\forall i \in I, \sum_{j \in A_{i}} \epsilon_{j} \leqslant \frac{1}{2} \inf _{x \in B_{n}} \epsilon\left(\phi_{i}(x)\right) .
$$

Let us make the convention to extend all functions which are compactly supported inside $B_{n}$, like $\left(g_{i} u\right) \circ \phi_{i}$ by the value 0 to the whole of $\mathbb{R}^{n}$. For each $i$, we choose a positive constant $a_{i}$ such that

$$
\left\|\left(g_{i} u\right) \circ \phi_{i}-J^{-a_{i}} \circ J^{+a_{i}}\left(\left(g_{i} u\right) \circ \phi_{i}\right)\right\|_{\infty}<\epsilon_{i} .
$$

Such a constant exists because the function $\left(g_{i} u\right) \circ \phi_{i}$ is uniformly continuous on $\mathbb{R}^{n}$. Since $T_{c}^{-} u$ is locally semiconcave, the function $\left(g_{i} T_{c}^{-} u\right) \circ \phi_{i}$, extended by zero outside of $B_{n}$, is semiconcave on $\mathbb{R}^{n}$ (see [Ber10]). We can assume by taking $a_{i}>0$ small enough that it is $a_{i}^{-1}$-semiconcave, so that

$$
\left[g_{i} u\right] \circ \phi_{i} \leqslant J^{-a_{i}} \circ J^{+a_{i}}\left(\left[g_{i} u\right] \circ \phi_{i}\right) \leqslant J^{-a_{i}} \circ J^{+a_{i}}\left(\left[g_{i} T_{c}^{-} u\right] \circ \phi_{i}\right)=\left[g_{i} T_{c}^{-} u\right] \circ \phi_{i}
$$

on $\mathbb{R}^{n}$. This implies in particular that the function $J^{-a_{i}} \circ J^{+a_{i}}\left(g_{i} u \circ \phi_{i}\right)$ is supported in $B_{n}$. As a consequence, the function $\left[J^{-a_{i}} \circ J^{+a_{i}}\left(g_{i} u \circ \phi_{i}\right)\right] \circ \phi_{i}^{-1}$, extended by zero outside of $\phi_{i}\left(B_{n}\right)$, is locally semiconcave on $M$, hence the function $S u$ is locally semiconcave, being a locally finite sum of locally semiconcave functions. By summation, we get

$$
u=\sum_{i \in I}\left(g_{i} u\right) \circ \phi_{i} \circ \phi_{i}^{-1} \leqslant S u \leqslant \sum_{i \in I}\left[g_{i} T_{c}^{-} u\right] \circ \phi_{i} \circ \phi_{i}^{-1}=T_{c}^{-} u,
$$

which, by Lemma 2.1, implies that $S u$ is a subsolution. We have $|u-S u|<\epsilon / 2$, by (5).

Next, we chose $b_{i}$ such that $\left[g_{i} T_{c}^{+} \circ T_{c}^{-} u\right] \circ \phi_{i}$ is $b_{i}^{-1}$-semiconvex, which implies that

$$
\begin{aligned}
{\left[g_{i} T_{c}^{+} \circ T_{c}^{-} u\right] \circ \phi_{i} } & =J^{+b_{i}} \circ J^{-b_{i}}\left(\left[g_{i} T_{c}^{+} \circ T_{c}^{-} u\right] \circ \phi_{i}\right) \\
& \leqslant J^{+b_{i}} \circ J^{-b_{i}}\left(\left[g_{i} u\right] \circ \phi_{i}\right) \leqslant J^{+b_{i}} \circ J^{-b_{i}}\left(\left[g_{i} S u\right] \circ \phi_{i}\right) \leqslant\left[g_{i} S u\right] \circ \phi_{i} .
\end{aligned}
$$

As above, this implies that $J^{+b_{i}} \circ J^{-b_{i}}\left(\left[g_{i} S u\right] \circ \phi_{i}\right)$ is supported on $B_{n}$. Note that it is also $C^{1,1}$ hence the function $\left(J^{+b_{i}} \circ J^{-b_{i}}\left(\left[g_{i} S u\right] \circ \phi_{i}\right)\right) \circ \phi_{i}^{-1}$, extended by zero outside of $\phi_{i}\left(B_{n}\right)$, is locally $C^{1,1}$ on $M$. By summation, we obtain that

$$
T_{c}^{+} \circ T_{c}^{-} u \leqslant \check{S} u \leqslant \check{S} \circ S u \leqslant S u \leqslant T_{c}^{-} u
$$

which implies that $\check{S} \circ S u$ is a subsolution. This function is locally $C^{1,1}$ as a locally finite sum of locally $C^{1,1}$ functions. Finally, we can assume by possibly reducing $b_{i}$ that

$$
\left\|\left(g_{i} S u\right) \circ \phi_{i}-J^{+b_{i}} \circ J^{-b_{i}}\left(\left(g_{i} S u\right) \circ \phi_{i}\right)\right\|_{\infty}<\epsilon_{i},
$$

which implies that $|\check{S} \circ S u-S u| \leqslant \epsilon / 2$ hence that $|\check{S} \circ S u-S u| \leqslant \epsilon$.

Theorem 7. We assume hypothesis 1. Let $\Omega \subset M$ be an open set and let $u$ be a continuous subsolution which is free on $\Omega$. Then the subsolution $u$ belongs to the closure, for the strong topology, of the set of $C^{1,1}$ subsolutions which are free on $\Omega$ and equal to $u$ on $\mathcal{A}_{u}$.

Proof. Let $\epsilon: M \rightarrow] 0, \infty)$ be a continuous function. We can chose $a_{i}$ and $b_{i}$ in such a way that $\check{S} \circ S u$ is a subsolution which is equal to $u$ on $\mathcal{A}_{u}$, and such that $|\check{S} \circ S u-u| \leqslant \epsilon$. However, $\check{S} \circ S u$ need not be free on $\Omega$. To preserve the freedom of $u$, we work with the modified cost

$$
\tilde{c}(x, y)=c(x, y)-\psi(y)
$$

where $\psi$ is a smooth bounded function such that $0 \leqslant \psi \leqslant \lambda_{u}$ (the leverage function of $u$ ), with strict inequalities on $\Omega$. The associated Lax-Oleinik operator is

$$
T_{\tilde{c}}^{-} v(x)=-\psi(x)+T_{c}^{-} v(x)
$$


Each subsolution for the $\operatorname{cost} \tilde{c}$ is thus a subsolution for the $\operatorname{cost} c$, and $\tilde{c}$ satisfies Hypothesis 1 . Moreover, the function $u$ is a subsolution for the cost $\tilde{c}$. We apply Theorem 6 and get a locally $C^{1,1}$ subsolution $w^{-}$for the cost $\tilde{c}$, which satisfies $\left|w^{-}-u\right| \leqslant \epsilon$ and $w^{-}=u$ on $\mathcal{A}_{u}$. This function then satisfies

$$
T_{c}^{-} w^{-}=\psi+T_{\tilde{c}}^{-} w^{-} \geqslant \psi+w^{-}
$$

hence it is a subsolution for the cost $c$. Similarly, by applying Theorem 6 with the modified cost $c(x, y)-\psi(x)$, we get a locally $C^{1,1}$ subsolution $w^{+}$(for the cost $c$ ) such that

$$
T_{c}^{+} w^{+} \leqslant w^{+}-\psi
$$

$\left|w^{+}-u\right| \leqslant \epsilon$ and $w^{+}=u$ on $\mathcal{A}_{u}$. We then set $w:=\left(w^{+}+w^{-}\right) / 2$ and claim that this locally $C^{1,1}$ subsolution is free on $\Omega$. This follows from the inequalities

$$
\begin{aligned}
& T_{c}^{-} w \geqslant\left(T_{c}^{-} w^{-}+T_{c}^{-} w^{+}\right) / 2 \geqslant w+\psi / 2, \\
& T_{c}^{+} w \leqslant\left(T_{c}^{+} w^{-}+T_{c}^{+} w^{+}\right) / 2 \leqslant w-\psi / 2,
\end{aligned}
$$

since $\psi$ is positive on $\Omega$. We also obviously have $|w-u| \leqslant \epsilon$ and $w=u$ on $\mathcal{A}_{u}$.

\section{$5 \quad$ Proof of Theorem 3}

We will build successively subsolutions $v_{1}, v_{2}, v_{3}$ which are all equal to $u$ on $\mathcal{A}_{u}$ and free on the complement $\Omega$ of $\mathcal{A}_{u}$. By Lemma 2.3, this also implies that the subsolutions $v_{i}$ are strict where $u$ is strict. We take

$$
v_{1}=\frac{T_{c}^{+} u+T_{c}^{+} \circ T_{c}^{-} u+T_{c}^{-} \circ T_{c}^{+} u+T_{c}^{-} u}{4},
$$

which is continuous, equal to $u$ on $\mathcal{A}_{u}$ and free on the complement of $\mathcal{A}_{u}$ by Lemma 2.2.

We then build $v_{2}$ by applying Theorem 7 to $v_{1}$, with $\Omega=M \backslash \mathcal{A}_{u}$, and get a locally $C^{1,1}$ subsolution $v_{2}$ which is free on $\Omega$ and equal to $u$ on $\mathcal{A}_{u}$.

The following mollification result, which will be proved in the Appendix using a procedure due to De Rham, allows to smooth our subsolution on $\Omega$.

Theorem 8. Let $f$ be a locally $C^{k, 1}$ function on $M$ and let $\epsilon: M \longrightarrow[0, \infty)$ be a continuous function. Then, there exists a locally $C^{k, 1}$ function $g: M \rightarrow \mathbb{R}$ which is smooth on the open set $\Omega:=\epsilon^{-1}(0,+\infty)$ and satisfies, for all $x \in M$,

$$
|f(x)-g(x)|+\left\|\mathrm{d}_{x} f-\mathrm{d}_{x} g\right\|+\cdots+\left\|\mathrm{d}_{x}^{k} f-\mathrm{d}_{x}^{k} g\right\| \leqslant \epsilon(x) .
$$

More precisely, we apply Theorem 8 to the function $f=v_{2}$, with $k=1$, and with a function $\epsilon(x)$ such that $\epsilon=0$ on $\mathcal{A}_{u}, \epsilon>0$ on $\Omega$ (the complement of $\mathcal{A}_{u}$ ), and $\epsilon \leqslant \lambda_{v_{2}}$ (the leverage function of $\left.v_{2}\right)$. We get a $C^{1,1}$ function $v_{3}$, which is smooth on $\Omega$ and is equal to $u$ on $\mathcal{A}_{u}$. Since $\left|v_{3}-v_{2}\right| \leqslant \lambda_{v_{2}}$, Lemma 2.5 implies that $v_{3}$ is a subsolution which is free on $\Omega$. Lemma 2.3 then implies that $v_{3}$ is strict where $u$ is strict.

\section{Proof of Theorem 4}

It is enough to prove the existence of a subsolution $u$ which is free on the complement of $\mathcal{A}$ and strict on the complement of $\overline{\mathcal{A}}$. Theorem 3 then implies the existence of a locally $C^{1,1}$ solution $v$ which is free and smooth on the complement of $\mathcal{A}$, and which is strict on the complement of $\overline{\mathcal{A}}$. We start with:

Lemma 6.1. If c satisfies Hypothesis 1 and admits a subsolution, then there exists a continuous subsolution $w_{1}$ which is free on the complement of $\mathcal{A}$. 
Proof. Let us consider a point $x \notin \mathcal{A}$. By definition, there exists a subsolution $v_{x}$ such that $x \notin \mathcal{A}_{v_{x}}$, hence, by Lemma 2.2, there exists a continuous subsolution $u_{x} \in \mathcal{S} \mathcal{S}_{C}$ which is free at $x$. By continuity of $u_{x}, T_{c}^{-} u_{x}$ and $T_{c}^{+} u_{x}$ we may consider a positive number $\epsilon_{x}$ and an open neighborhood of $x, O_{x}$, on which the following holds:

$$
\forall y \in O_{x}, \quad T_{c}^{-} u_{x}(y)-\epsilon_{x}>u_{x}(y)>T_{c}^{+} u_{x}(y)+\epsilon_{x} .
$$

The set $M \backslash \mathcal{A}$ satisfies the Lindelöf property (it is a separable metric space). We can thus extract a countable covering $O_{n}, n \in \mathbb{N}$ of the covering $O_{x}, x \in M \backslash \mathcal{A}$. Denoting by $u_{n}$ and $\epsilon_{n}$ the continuous subsolution and positive real number associated to $O_{n}$, we consider a convex combination

$$
w_{1}=\sum_{n \in \mathbb{N}} a_{n} u_{n}
$$

where $a_{n}$ is a sequence of positive numbers such that $\sum_{\mathbb{N}} a_{n}=1$ and such that the sum in the definition of $w_{1}$ is normally convergent on each compact set. The function $w_{1}$ is then a continuous subsolution. For each $x \notin \mathcal{A}$, there exists $n_{0} \in \mathbb{N}$ such that $x \in O_{n_{0}}$, and we have

$$
T_{c}^{-} w_{1}(x)=T_{c}^{-}\left(\sum_{n \in \mathbb{N}} a_{n} u_{n}\right)(x) \geqslant \sum_{n \in \mathbb{N}} a_{n} T_{c}^{-} u_{n}(x) \geqslant a_{n_{0}} \epsilon_{n_{0}}+\sum_{n \in \mathbb{N}} a_{n} u_{n}>w_{1}(x) .
$$

A similar computation shows that $T_{c}^{+} w_{1}(x)<w_{1}(x)$.

Lemma 6.2. If there exists a continuous subsolution, then there exists a continuous subsolution $w_{2}$ which is strict at each pair $(x, y)$ where a strict continuous subsolution exists. Under Hypothesis 1 , the subsolution $w_{2}$ is then strict outside of $\overline{\mathcal{A}}$.

Proof. Since $M$ is separable, the set $\mathcal{S S}_{C}$ of continuous subsolutions is also separable (for the compact-open topology), and we consider a dense subsequence $\left(u_{n}\right)_{n \in \mathbb{N}}$. Set

$$
w_{2}=\sum_{n \in \mathbb{N}} a_{n} u_{n}
$$

where the $a_{n}$ are positive real numbers such that $\sum a_{n}=1$ and the sum (6) is uniformly convergent on each compact subset. The function $w_{2}$ is a subsolution since it is a convex combination of subsolutions. If now $(x, y) \in \overline{\mathcal{A}}_{w_{2}}$, summing the inequalities

$$
\forall n \in \mathbb{N}, \quad a_{n}\left(u_{n}(y)-u_{n}(x)\right) \leqslant a_{n} c(x, y),
$$

gives an equality, therefore all inequalities are equalities and

$$
\forall n \in \mathbb{N}, \quad(x, y) \in \overline{\mathcal{A}}_{u_{n}} .
$$

By density of the sequence $u_{n}$, we deduce that $(x, y) \in \overline{\mathcal{A}}_{u}$ for each continuous solution $u$. Under Hypothesis $1, \overline{\mathcal{A}}$ is exactly the set of pairs at which no continuous subsolution is strict: $\overline{\mathcal{A}}=\bigcap_{u \in \mathcal{S} \mathcal{S}_{C}} \overline{\mathcal{A}}_{u}$ hence, $(x, y) \in \overline{\mathcal{A}}$.

To finish the proof of Theorem 4, we consider the subsolution $u=\left(w_{1}+w_{2}\right) / 2$. This subsolution is free on the complement of $\mathcal{A}$ because $w_{1}$ is, and it is strict on the complement of $\overline{\mathcal{A}}$ because $w_{2}$ is. 


\section{A Proof of Theorem 8}

We prove Theorem 8 using a regularization procedure due to De Rham, see [dR73]. The idea of De Rham is to construct an action $\mathfrak{t}$ of $\mathbb{R}^{n}$ on $\mathbb{R}^{n}$ by smooth diffeomorphisms supported on the unit sphere $B_{n}$, in such a way that the induced action on $B_{n}$ is conjugated to the standard action of $\mathbb{R}^{n}$ on itself by translations. More precisely, there exists a diffeomorphism $\mathfrak{h}: B_{n} \longrightarrow \mathbb{R}^{n}$ and diffeomorphisms $\mathfrak{t}_{y}$, $y \in \mathbb{R}^{n}$, of $\mathbb{R}^{n}$, equal to the identity outside of the open unit ball $B_{n}$, such that the map $(x, y) \longmapsto \mathfrak{t}_{y}(x)$ is smooth and such that

$$
\mathfrak{h} \circ \mathfrak{t}_{y}=y+\mathfrak{h}
$$

on $B_{n}$. This implies that $\mathfrak{t}$ is an action of the group $\mathbb{R}^{n}$ on $\mathbb{R}^{n}$, which means that $\mathfrak{t}_{y} \circ \mathfrak{t}_{y^{\prime}}=\mathfrak{t}_{y+y^{\prime}}$ for each $y, y^{\prime}$. Since $\mathfrak{t}$ is smooth, $\mathfrak{t}_{0}=I d$, and $\mathfrak{t}_{y}=I d$ outside of the unit ball, the maps $\mathfrak{t}_{y}$ converge uniformly to the identity as $y \longrightarrow 0$, and all their derivatives converge uniformly to the derivatives of the identity.

Let us give some details on the construction of $\mathfrak{h}$ and $\mathfrak{t}$. We set

$$
\mathfrak{h}(x)=\frac{h(\|x\|)}{\|x\|} x,
$$

where $h:\left[0,1\left[\rightarrow \mathbb{R}_{+}\right.\right.$is a smooth, strictly increasing $\left(h^{\prime}>0\right)$ function such that

$$
\begin{cases}h(r)=r, & 0 \leqslant r \leqslant 1 / 3 \\ h(r)=\exp \left((r-1)^{-2}\right), & 2 / 3 \leqslant r<1\end{cases}
$$

We then define $\mathfrak{t}_{y}$, for each $y \in \mathbb{R}^{n}$ by

$$
\begin{cases}\mathfrak{t}_{y}(x)=\mathfrak{h}^{-1}(\mathfrak{h}(x)+y) & \text { if } x \in B_{n} \\ \mathfrak{t}_{y}(x)=x & \text { if } x \in \mathbb{R}^{n} \backslash B_{n} .\end{cases}
$$

It is clear from these formula that $\mathfrak{t}_{y+y^{\prime}}=\mathfrak{t}_{y} \circ \mathfrak{t}_{y^{\prime}}$. The only issue is the smoothness of $\mathfrak{t}$. Differentiating the previous group property with respect to $y^{\prime}$ and taking $y^{\prime}=0$ yields the following relation:

$$
\frac{\partial}{\partial y} \mathfrak{t}_{y}=\frac{\partial}{\partial y} \mathfrak{t}_{0} \circ \mathfrak{t}_{y}
$$

This implies that

$$
\mathfrak{t}_{y}(x)=x+\int_{0}^{1} \frac{\mathrm{d}}{\mathrm{d} t} \mathfrak{t}_{t y}(x) \mathrm{d} t=x+\int_{0}^{1}\left(\frac{\partial}{\partial y} \mathfrak{t}_{t y}(x)\right) y \mathrm{~d} t=x+\int_{0}^{1}\left(\frac{\partial}{\partial y} \mathfrak{t}_{0}\left(\mathfrak{t}_{t y}(x)\right)\right) y \mathrm{~d} t .
$$

In other words, the map $\boldsymbol{t}_{y}$ is the time-one flow of the vector field $X_{y}(x):=M(x) y$, where $M(x)=$ $\partial_{y} \mathfrak{t}_{y}(x)_{\mid y=0}$. In order to prove that the map $\mathfrak{t}$ is smooth, it is enough to observe that the matrix $M(x)$ depends smoothly on $x$. This matrix can be computed, recalling that the gradient of the norm $x \mapsto\|x\|$ is $r_{x}:=x /\|x\|:$

$$
M(x)=\mathrm{d}_{\mathfrak{h}(x)} \mathfrak{h}^{-1}=\frac{1}{h^{\prime}(\|x\|)} r_{x}^{t} r_{x}+\frac{\|x\|}{h(\|x\|)}\left(I_{n}-r_{x}^{t} r_{x}\right) .
$$

Since $1 / h, 1 / h^{\prime}$, as well as all their derivatives go to 0 when $\|x\| \rightarrow 1$, we conclude that $M(x)$ is smooth.

We have exposed the construction of $\mathfrak{h}$ and $\mathfrak{t}$. They allow to define a local regularization procedure with the help of a smooth kernel $K_{1}: \mathbb{R}^{n} \rightarrow[0, \infty)$. We assume that $K_{1}$ is supported in the unit ball $B_{n}$, and that $\int K_{1}=1$. For $\eta>0$, we set $K_{\eta}(x)=\eta^{-n} K_{1}\left(\eta^{-1} x\right)$.

Lemma A.1. Let $O \subset \mathbb{R}^{n}$ be an open set containing $\bar{B}_{n}$. Given a locally integrable function $f: O \longrightarrow$ $\mathbb{R}$ and $\eta \in] 0,1[$, we define

$$
f_{\eta}(x)=\int_{\mathbb{R}^{n}} f\left(\mathfrak{t}_{y}(x)\right) K_{\eta}(-y) \mathrm{d} y
$$

The following assertions hold: 
1. The function $f_{\eta}$ is $C^{\infty}$ in $B_{n}$, and equal to $f$ outside of $B_{n}$,

2. If $f$ is $C^{k}$ on $O$, then so are the functions $f_{\eta}$, and $f_{\eta} \longrightarrow f$ in $C^{k}$ as $\eta \longrightarrow 0$.

3. If $f$ is $C^{k, 1}$ on $O$, then so are the functions $f_{\eta}$, and $\lim \sup _{\eta \rightarrow 0} \operatorname{Lip}\left(\mathrm{d}^{k} f_{\eta}\right) \leqslant \operatorname{Lip}\left(\mathrm{d}^{k} f\right)$.

4. If, in some open set $O^{\prime} \subset O, f$ is $C^{l}$ in $O^{\prime}$, then so is $f_{\eta}$.

Proof. On $B_{n}$ we have

$$
f_{\eta} \circ \mathfrak{h}^{-1}=\left(f \circ \mathfrak{h}^{-1}\right) \star K_{\eta},
$$

where $\star$ is the convolution. Since the functions $K_{\eta}$ are smooth, this implies the first claim. Writing

$$
f_{\eta}-f=\int_{B(0, \eta)}\left(f \circ \mathfrak{t}_{y}-f\right) K_{\eta}(-y) \mathrm{d} y
$$

and observing that $f \circ \mathfrak{t}_{y}-f \longrightarrow 0$ in $C^{k}\left(\mathbb{R}^{n}, \mathbb{R}^{n}\right)$ as $y \longrightarrow 0$ (because $\mathfrak{t}_{y} \longrightarrow I d$ in $C^{k}\left(\mathbb{R}^{n}, \mathbb{R}^{n}\right)$ ) yields the second claim. We will now prove that

$$
\limsup _{y \rightarrow 0} \operatorname{Lip}\left(\mathrm{d}^{k}\left(f \circ \mathfrak{t}_{y}\right)\right) \leqslant \operatorname{Lip}\left(\mathrm{d}^{k} f\right)
$$

which yields the third claim in view of the relation

$$
\mathrm{d}_{x}^{k} f_{\eta}=\int_{B(0, \eta)} \mathrm{d}_{x}^{k}\left(f \circ \mathfrak{t}_{y}\right) K_{\eta}(-y) \mathrm{d} y .
$$

Let us consider a component $\partial_{x}^{\alpha}\left(f \circ \mathfrak{t}_{y}\right)$ of the differential $\mathrm{d}^{k}\left(f \circ \mathfrak{t}_{y}\right)$, where $\alpha=\left(\alpha_{1}, \ldots, \alpha_{n}\right)$ is a multi-index such that $|\alpha|=\sum \alpha_{i}=k$. By the Faà di Bruno formula, expressed in terms of partial differentials (see [CS96] for example), we have

$$
\partial_{x}^{\alpha}\left(f \circ \mathfrak{t}_{y}\right)=\sum_{1 \leqslant|\lambda| \leqslant|\alpha|} \partial_{\mathfrak{t}_{y}(x)}^{\lambda} f \cdot B_{\alpha, \lambda}\left(\mathrm{d}_{x} \mathfrak{t}_{y}, \ldots, \mathrm{d}_{x}^{|\alpha|} \mathfrak{t}_{y}\right)
$$

where the $B_{\alpha, \lambda}$ are universal multi-variable polynomials with no constant terms. These polynomials satisfy the equalities

$$
B_{\alpha, \alpha}(I d, 0, \cdots, 0)=1 \quad \text { and } \quad B_{\alpha, \lambda}(I d, 0, \cdots, 0)=0
$$

for all $\lambda \neq \alpha$. Since $\mathfrak{t}_{y} \longrightarrow I d$ in $C^{\infty}$, the first of these equalities implies that the function $x \longmapsto$ $B_{\alpha, \alpha}\left(\mathrm{d}_{x} \mathfrak{t}_{y}, \ldots, \mathrm{d}_{x}^{|\alpha|} \mathfrak{t}_{y}\right)$ is converging to 1 in $C^{\infty}$. Concerning the other factor in this term, we have

$$
\operatorname{Lip}\left(\left(\partial^{\alpha} f\right) \circ \mathfrak{t}_{y}\right) \leqslant \operatorname{Lip}\left(\partial^{\alpha} f\right) \operatorname{Lip}\left(\mathfrak{t}_{y}\right) \longrightarrow \operatorname{Lip}\left(\partial^{\alpha} f\right) .
$$

We deduce that the upper limit of the Lipschitz constants of the term corresponding to $\lambda=\alpha$ is not greater than $\operatorname{Lip}\left(\partial^{\alpha} f\right)$.

On the other hand, for each of the terms with $\lambda \neq \alpha$, the function $x \longmapsto B_{\alpha, \lambda}\left(\mathrm{d}_{x} \mathfrak{t}_{y}, \ldots, \mathrm{d}_{x}^{|\alpha|} \mathfrak{t}_{y}\right)$ is converging to 0 in $C^{\infty}$ hence the Lipschitz constant of the function

$$
x \longmapsto \partial_{\mathfrak{t}_{y}(x)}^{\lambda} f \cdot B_{\alpha, \lambda}\left(\mathrm{d}_{x} \mathfrak{t}_{y}, \ldots, \mathrm{d}_{x}^{|\alpha|} \mathfrak{t}_{y}\right)
$$

is converging to 0 . We conclude that

$$
\lim \sup \operatorname{Lip}\left(\partial^{\alpha}\left(f \circ \mathfrak{t}_{y}\right)\right) \leqslant \operatorname{Lip}\left(\partial^{\alpha} f\right),
$$

which implies (7) hence the third point of the statement.

Regarding the last claim of the statement, we consider the set $\Omega:=\cap_{y \in \bar{B}(0, \eta)} \mathfrak{t}_{y}^{-1}\left(O^{\prime}\right)$, and claim that $\Omega$ is open. Assuming the claim, we observe that the function $f_{\eta}$ is smooth in $B_{n}$ and that it is 
$C^{l}$ in $\Omega$. Since the maps $\mathfrak{t}_{y}$ are all the identity outside of $B_{n}$, the set $\Omega$ contains $O^{\prime}-B_{n}$. We have covered $O^{\prime}$ by two open sets, $B_{n}$ and $\Omega$, such that the $f_{\eta}$ is $C^{l}$ on each of them, we conclude that this function is $C^{l}$ on $O^{\prime}$.

To prove that $\Omega$ is open, we fix $x_{0} \in \Omega$. For each $y_{0} \in \bar{B}(0, \eta)$, we have $\mathfrak{t}_{y_{0}}\left(x_{0}\right) \in O^{\prime}$, hence there exists an open set $U_{y_{0}}$ containing $y_{0}$ and an open set $\Omega_{y_{0}}$ containing $x_{0}$ such that $\mathfrak{t}_{y}(x) \in O^{\prime}$ far all $(x, y) \in \Omega_{y_{0}} \times U_{y_{0}}$. By compactness, there exists finitely many points $y_{i} \in \bar{B}(0, \eta)$ such that the open sets $U_{y_{i}}$ cover $\bar{B}(0, \eta)$. The open intersection $\cap \Omega_{y_{i}}$, which contains $x_{0}$, is then contained in $\Omega$. Since this holds for each $x_{0} \in \Omega$, we have proved that $\Omega$ is open.

Lemma A.2. Let $O$ be open subsets of $\mathbb{R}^{n}$ and let $f: O \rightarrow \mathbb{R}$ be a $C^{k, 1}$ function. Given a continuous function $\epsilon: O \rightarrow[0, \infty)$, there exists a function $f_{\epsilon}$ such that:

1. the function $f_{\epsilon}$ is $C^{\infty}$ in the open set $\{x \in O, \epsilon(x)>0\} \subset O$,

2. $\left|f_{\epsilon}(x)-f(x)\right|+\left\|\mathrm{d}_{x} f_{\epsilon}-\mathrm{d}_{x} f\right\|+\cdots+\left\|\mathrm{d}_{x}^{k} f_{\epsilon}-\mathrm{d}_{x}^{k} f\right\| \leqslant \epsilon(x)$ for each $x \in O$,

3. the function $f_{\epsilon}$ is $C^{k, 1}$ on $O$, and $\operatorname{Lip}\left(\mathrm{d}^{k} f_{\epsilon}\right) \leqslant 1+\operatorname{Lip}\left(\mathrm{d}^{k} f\right)$.

Proof. Let us denote by $F$ the closed set $\{\epsilon=0\}$. The complement of $F$ in $O$ is open, and we consider a locally finite covering $\left(O_{i}\right)_{i \in \mathbb{N}^{*}}$ of $O \backslash F$ by open balls compactly included in $O \backslash F$. Since $\inf \left\{\epsilon(x), \quad x \in O_{i}\right\}>0$. we can construct inductively, using Lemma A.1 a sequence of functions, $\left(f_{i}\right)_{i \in \mathbb{N}}$ such that

- $f_{0}=f$,

- for each $i \in \mathbb{N}$, the function $f_{i+1}$ is $C^{\infty}$ in $O_{1} \cup \cdots \cup O_{i+1}$,

- for each $i \in \mathbb{N}$, the functions $f_{i}$ and $f_{i+1}$ are equal in $O \backslash O_{i+1}$,

- for each $i \in \mathbb{N}$, the function $f_{i+1}$ is $C^{k, 1}$ in $O$, and $\operatorname{Lip}\left(\mathrm{d}^{k} f_{i+1}\right) \leqslant 2^{-i-1}+\operatorname{Lip}\left(\mathrm{d}^{k} f_{i}\right)$,

- $\left|f_{i+1}(x)-f_{i}(x)\right|+\left\|\mathrm{d}_{x} f_{i+1}-\mathrm{d}_{x} f_{i}\right\|+\cdots+\left\|\mathrm{d}_{x}^{k} f_{i+1}-\mathrm{d}_{x}^{k} f_{i}\right\| \leqslant 2^{-1-i} \epsilon(x)$ for each $x \in O, i \in \mathbb{N}$,

Each point of $O$ has a neighborhood on which the sequence $f_{i}$ is eventually constant, hence the limit $f_{\epsilon}:=\lim f_{i}$ is well-defined and smooth on $\cup_{i} O_{i}=O \backslash F$. The desired estimates on $f_{\epsilon}$ follow immediately from the inductive estimates by summation.

Proof of Theorem 8. We fix a locally finite atlas $\left(\phi_{i}\right)_{i \in \mathbb{N}^{*}}$ constituted of smooth maps $\phi_{i}$ : $2 B_{n} \rightarrow M$, where $B_{n}$ is the open unit ball. We assume that all the images $\phi_{i}\left(2 B_{n}\right), i \in \mathbb{N}^{*}$ are relatively compact in $M$ and that the $\phi_{i}\left(B_{n}\right), i \in \mathbb{N}^{*}$ still cover $M$. By Lemma A.2, it is possible to construct inductively a sequence of functions $f_{i}$, by iteratively modifying $f_{i} \circ \phi_{i+1}$ on $B_{n}$, such that

- $f_{0}=f$,

- for each $i \in \mathbb{N}$, the function $f_{i+1}$ is $C^{\infty}$ in $\bigcup_{j \leqslant i+1} \phi_{j}\left(B_{n}\right) \cap \Omega$,

- for each $i \in \mathbb{N}$, in $M \backslash \phi_{i+1}\left(B_{n}\right)$, the functions $f_{i}$ and $f_{i+1}$ are equal,

- for each $i \in \mathbb{N}$, the function $f_{i+1}$ is $C^{k, 1}$ on $M$,

- for each $i \in \mathbb{N}, x \in M,\left|f_{i}(x)-f_{i+1}(x)\right|+\cdots+\left\|\mathrm{d}_{x}^{k} f_{i}-\mathrm{d}_{x}^{k} f_{i+1}\right\| \leqslant 2^{-i-1} \epsilon(x)$.

Each point $x \in M$ has a neighborhood on which the sequence $f_{i}$ is eventually constant, hence the $\operatorname{limit} g=\lim f_{i}$ is well defined, locally $C^{k, 1}$, and smooth on $\Omega$. The inequality on the differentials follows by summation from the iterative assumptions. 


\section{References}

[AD00] L. Ambrosio and N. Dancer. Calculus of variations and partial differential equations. Springer-Verlag, Berlin, 2000. Topics on geometrical evolution problems and degree theory, Papers from the Summer School held in Pisa, September 1996, Edited by G. Buttazzo, A. Marino and M. K. V. Murthy.

[BB07] P. Bernard and B. Buffoni. Weak KAM pairs and Monge-Kantorovich duality. In Asymptotic analysis and singularities - elliptic and parabolic PDEs and related problems, volume 47 of Adv. Stud. Pure Math., pages 397-420. Math. Soc. Japan, Tokyo, 2007.

[Ber02] P. Bernard. Connecting orbits of time dependent Lagrangian systems. Annales de l'institut Fourier, 52(5):1533-1568, 2002.

[Ber07] P. Bernard. Existence of $C^{1,1}$ critical sub-solutions of the Hamilton-Jacobi equation on compact manifolds. Ann. Sci. École Norm. Sup. (4), 40(3):445-452, 2007.

[Ber08] P. Bernard. The dynamics of pseudographs in convex Hamiltonian systems. J. Amer. Math. Soc., 21(3):615-669, 2008.

[Ber10] P. Bernard. Lasry-Lions regularization and a lemma of Ilmanen. Rend. Semin. Mat. Univ. Padova, 124:221-229, 2010.

[BR05] P. Bernard and J.M. Roquejoffre. Convergence to time-periodic solutions in time-periodic Hamilton-Jacobi equations on the circle. Communications in Partial Differential Equations, 29(3-4):457-469, 2005.

[Car01] P. Cardaliaguet. Front propagation problems with nonlocal terms. II. J. Math. Anal. Appl., 260(2):572-601, 2001.

[CISM00] G. Contreras, R. Iturriaga, and H. Sanchez-Morgado. Weak solutions of the HamiltonJacobi equation for time periodic Lagrangians. preprint, 2000.

[CS96] G. M. Constantine and T. H. Savits. A multivariate Faà di Bruno formula with applications. Trans. Amer. Math. Soc., 348(2):503-520, 1996.

[dR73] G. de Rham. Variétés différentiables. Formes, courants, formes harmoniques. Hermann, Paris, 1973. Troisième édition revue et augmentée, Publications de l'Institut de Mathématique de l'Université de Nancago, III, Actualités Scientifiques et Industrielles, No. $1222 \mathrm{~b}$.

[Fat98] A. Fathi. Sur la convergence du semi-groupe de Lax-Oleinik. Comptes Rendus de l'Académie des Sciences-Series I-Mathematics, 327(3):267-270, 1998.

[FM00] A. Fathi and J. Mather. Failure of convergence of the Lax-Oleinik semi-group in the timeperiodic case. Bull. Soc. Math. France, 128(3):473-483, 2000.

[FS04] A. Fathi and A. Siconolfi. Existence of $C^{1}$ critical subsolutions of the Hamilton-Jacobi equation. Invent. Math., 155(2):363-388, 2004.

[FZ10] A. Fathi and M. Zavidovique. Ilmanen's lemma on insertion of $C^{1,1}$ functions. Rend. Semin. Mat. Univ. Padova, 124:203-219, 2010.

[Gom05] D.A. Gomes. Viscosity solution method and the discrete Aubry-Mather problem. Discrete and Continuous Dynamical Systems, Series A, 13:103-116, 2005.

[Hir94] M. W. Hirsch. Differential topology, volume 33 of Graduate Texts in Mathematics. SpringerVerlag, New York, 1994. Corrected reprint of the 1976 original. 
[Ilm93] T. Ilmanen. The level-set flow on a manifold. In Differential geometry: partial differential equations on manifolds (Los Angeles, CA, 1990), volume 54 of Proc. Sympos. Pure Math., pages 193-204. Amer. Math. Soc., Providence, RI, 1993.

[Zav10] M. Zavidovique. Existence of $C^{1,1}$ critical subsolutions in discrete weak KAM theory. $J$. Mod. Dyn., 4(4):693-714, 2010.

[Zav12] M. Zavidovique. Strict subsolutions and Mañé potential in discrete weak KAM theory. Commentarii Mathematici Helvetici, 87(1):1-39, 2012. 